\title{
Professional Competence in Nursing: A Hybrid Concept Analysis
}

\author{
Leila Valizadeh ${ }^{1}$, Vahid Zamanzadeh ${ }^{2}$, Maghsoud Eskandari ${ }^{3}$ and Samaneh Alizadeh ${ }^{4,}$ \\ ${ }^{1}$ Department of Pediatric Nursing, Faculty of Nursing and Midwifery, Tabriz University of Medical Sciences, Tabriz, Iran \\ ${ }^{2}$ Department of Medical-Surgical Nursing, Faculty of Nursing and Midwifery, Tabriz University of Medical Sciences, Tabriz, Iran \\ ${ }^{3}$ Faculty of Medical Sciences, Imam Khomeini Research, Treatment and Educational Center, Sarab, Iran \\ ${ }^{4}$ Department of Medical-Surgical Nursing, Student Research Committee, Faculty of Nursing and Midwifery, Tabriz University of Medical Sciences, Tabriz, Iran \\ "Corresponding author: Department of Medical-Surgical Nursing, Student Research Committee, Faculty of Nursing and Midwifery, Tabriz University of Medical Sciences, \\ Tabriz, Iran. Tel: +98-9144199354, Email: alizadehsamaneh016@gmail.com \\ Received 2019 February 12; Accepted 2019 June 18.
}

\begin{abstract}
Objectives: Nurses, as the largest group providing health care services, play a critical role in the continuity of care and respond to services commensurate with their competence. Professional competence in nursing is a complex and ambiguous concept and needs the redefinition of competence and development of its definition and concept. In this study, we aimed to conceptually analyze professional competence in nursing.

Methods: In this study, a hybrid concept analysis was used. In the literature review phase, the concept of 'competence' was investigated through valid databases limited to 2000 - 2018 and the participants' experiences also appeared in the fieldwork phase. In the fieldwork phase, the participants revealed some aspects of professional competence in nursing, which were not well-addressed in the literature. Finally, the comprehensive definition of professional competence in nursing was presented after integrating the findings obtained in these two phases.

Results: According to the findings, professional competence in nursing is a combined, complex, multidimensional, and contextdependent concept, and consists of different domains. Based on the analysis results of the fieldwork phase, this concept was classified into 16 domains and the participants largely contributed to the provision of a comprehensive definition based on the definitions of professional competence presented in the literature.

Conclusions: Based on the domains of professional competence in nursing obtained from this study, an appropriate instrument can be developed to assess professional competence in nursing for students and nurses.
\end{abstract}

Keywords: Professional Competence, Nursing, Qualitative Research

\section{Background}

One of the consequences of development in a society is task specialization in a large number of human life areas, including the ones relevant to the provision of healthcare services. Thus individuals who want to receive quality services specifically concern the competency (1). Nurses, as the largest group of health care services, provide health care to patients. The significance and impact of these services on the quality of health care and achievement of health goals are publically known (2) and nurses should operate proportional to the changes in systems providing health services. To this end, the detection of key issues affecting the nursing profession is of paramount importance (3).

One of these issues, which has become a hotbed of debate in many countries around the world, is nurses' competence. In order to improve the quality of nursing care, employers in this field prefer to employ competent nurses to ensure that help seekers are provided with quality care (4). Today, nursing is considered a complicated profession requiring a variety of skills. As a result, the nurses are expected to further expand their abilities to adapt to new technologies, new roles, new workplaces, and new health challenges. In other words, nurses should increasingly improve their professional competence (5). Professional competence develops and is achieved over time. In fact, the acquisition of competence is a process, not an endpoint (6).

In Iran, there has been a growing concern about the nurses' competence over the recent years since health service delivery systems are inevitably obliged to enhance the effectiveness of their human resources due to the increased community awareness and expectations to receive high-quality services (7).

Differences in viewpoints regarding the type and nature of the roles, expertise, and various clinical environments, as well as the multiplicity of nurses' tasks and ex- 
pectations of nurses, have caused complexities in defining the term of competence in nursing during the 21st century (8). This necessitates the redefinition of competence, the development of its definition and concept, as well as further evaluation of the term, to define the attributes of competent nurses more precisely.

The review of the literature shows that a majority of studies published in the field of professional competence have been quantitative, though, quantitative studies fail to delve into this concept since competence is a combined, complex, multidimensional, and context-dependent concept. Hence, the concept analysis requires a close examination, which is mainly possible via qualitative studies. In this study, the researchers were to adopt a hybrid model containing the findings obtained from theoretical analysis and fieldwork phase to overcome the ambiguities in this field. This, in turn, provides an opportunity to develop accurate instruments to evaluate professional competence and employ competent nurses in health care systems. Also, in the review of literature, concept analysis study of the competency in nursing by Bagheri Nesami et al. (9) was found.

\section{Objectives}

Owing to changes in the health system and new expectations of nurses, a new definition of competency is felt, thus in this study, we aimed to provide a comprehensive and new definition of professional competence in nursing by strengthening previous studies.

\section{Methods}

In this study, the Swartz-Barcott and Kim's method or hybrid model was used to analyze the concept of professional competence in nursing, because nurses', instructors, and nursing authorities' viewpoints make the definition more precise and comprehensive. This method is a hybrid concept development model and includes a threephase process (namely theoretical phase, fieldwork phase, final analytical phase). In the theoretical phase, the concept is selected, a thorough review of the literature is carried out, and a working definition is selected. This phase aims to determine how the concept is defined, used, and measured in the relevant literature. In the fieldwork phase, the concept is corroborated and refined by collecting qualitative information from participants. In the final analysis phase, the data extracted from two previous phases are compared to finally provide a comprehensive definition of the concerned concept (10).

\subsection{Ethical Consideration}

After obtaining the ethical approval from the Ethics Committee of Tabriz University of Medical Sciences (approval No. 5/D/108503), written informed consent, and permission to record the interviews was obtained from all participants. The study's purpose and method were explained to the participants. They were informed that their contribution in the study was voluntary and the withdrawal was possible at any time.

\subsection{Theoretical Phase}

The concept 'professional competence in nursing' was looked up in ProQuest, Google Scholar, Science Direct, PubMed, Ovid, Medline, and CINAHL databases as well as Persian-language databases, including Magiran and SID. The keywords used in the search process were "competence", "competency", "nursing competency and competence", "professional competency and competence", and the search findings were limited to 2000 - 2018. In the preliminary review, 155 articles were found. With a close examination of the articles and removing irrelevant and repetitive articles based on the objective of the study, 12 full-text articles were included (Box 1 ).

\begin{tabular}{l}
\hline Box 1. Resource Selection Process in the Theoretical Phase \\
\hline A Preliminary Search of Resources (155 Studies) \\
\hline Deleted resources (143 studies) \\
Repetitive resources ( 35 studies) \\
Unavailability of full text (6 studies) \\
Irrelevant resources (102 studies) \\
Selected resources (12 studies) \\
\hline
\end{tabular}

\subsection{Fieldwork Phase}

Qualitative research is a comprehensive and in-depth study of a phenomenon by collecting and providing rich narrative information by using a flexible method (11). The use of a focused group to collect information is a valuable method for qualitative researchers. The focused group is a semi-structured group meeting conducted by a group leader in an informal context to gather information about a specific topic (12). In this way, individuals are sources of valuable information and the group dynamism encourages individuals to describe their viewpoints, which are less likely to be raised in in-person interviews (13). Regarding the purpose of the current study, a focused group meeting was held at the conference hall of Tabriz University of Medical Sciences for 2 hours with the participation of 15 subjects and the presence of the head of the Nursing Department, the head of the Nursing and Midwifery Faculty 
of Tabriz University of Medical Sciences, a professional expert in the field of competence, and several managers of nursing services, experienced head nurses, nurses, and faculty members. The meeting leader was the researcher.

In order to reach more details, four members of the Faculty of Nursing and Midwifery who were selected by using purposive sampling took part in in-depth semistructured interviews. The main question in the interviews was as follows: "What is professional competence in nursing?" Further questions (e.g., who is a competent nurse? What are the competency benchmarks in nursing? And so on) were asked to explore the concept in more details. The interviews were conducted in the professors' offices, and each interview lasted for 30 - 40 minutes, the interviews were recorded and then transcribed verbatim. Data analysis started at the same time with the data collection procedure using the conventional content analysis method. In the following, while coding the data, the themes gradually were extracted. The interviews continued until data saturation was reached. To observe confidentiality of information, each participant was assigned a number and the quotes were reported based on the numbers.

\subsection{Final Analytical Phase}

In this phase, the findings from the two previous phases were merged, and then a comprehensive definition of professional competence in nursing was presented.

\section{Results}

\subsection{Theoretical Phase}

\subsubsection{Literature Review}

The review of the literature revealed a number of definitions proposed for competence from different perspectives; however, no common definition for this concept was found. Moreover, the results of studies show that some concepts such as competence, efficiency, ability, performance, and skill have been interchangeably used in articles and that the concepts need to be explicitly clarified.

In nursing and other health-relevant professions, as Pai claims, the complexity and diversity of the abilities needed to provide proper care, as well as the variety and breadth of care provision environments, make it difficult to provide a single definition of competence (14).

In examining the qualifications associated with competence, it is necessary to consider practical knowledge, experience, expertise in care, communication, environment, motivation, and professionalization. Furthermore, the majority of definitions of competence presented in the literature consist of three main components (i.e., knowledge, attitudes and skills). One remarkable point in all these definitions is their evolution from partial to holistic point of view (15).

In all papers, knowledge has been considered the most important prerequisite of competence. It is also pointed out that knowledge cannot guarantee competence; however, competence cannot be achieved without knowledge and awareness. In previous studies, skill is also another prerequisite for competence (16).

The consequences of competence can be discussed in three aspects: professional, personal, and organizational (15). In the professional aspect, competence enables an individual to play his expected care role. In the personal aspect, competence is a factor affecting the nurses' job satisfaction and preventing them to quit their job. Lack of competence is also regarded as one of the main causes of dissatisfaction among nurses. In the organizational aspect, the availability of competent staff not only reduces the costs but also is associated with the use of available resources and facilities (17).

Other definitions of the concept are presented in Table 1.

\subsection{Fieldwork Phase}

Almost all participants of the study believed that competence is a combination of different factors.

Sixteen domains were extracted from the findings in this phase (Box 2).

\begin{tabular}{l}
\hline Box 2. Domains of Professional Competence in Nursing \\
\hline Domains \\
\hline Knowledge and awareness \\
\hline Skill \\
\hline Attitude \\
\hline Experience \\
\hline Motivation \\
\hline Clinical judgment \\
\hline Professional independence \\
\hline Relationship with patients \\
\hline Holistic care \\
\hline Teamwork and cooperation \\
\hline Management and leadership \\
\hline Moral action \\
\hline Perseverance and persistence \\
\hline Ability to work in difficult and complex situations \\
\hline Creativity \\
\hline Responsibility \\
\hline
\end{tabular}


Each theme is briefly explained by referring to the participants' quotations.

\subsubsection{Knowledge and Awareness}

According to one of the participants, "One of the major components of competence is professional knowledge and awareness. Nursing knowledge is a key component in this field. Otherwise, an individual holding a diploma can wellperform nursing operations after taking a training course. What distinguishes nurses from others is knowledge and awareness and knowing how to use them in different situations."

\subsubsection{Skill}

Given the significance of skills in professional competence, one participant mentioned, "Nurses' skills may form the major part of their competence since an unskilled nurse cannot attract patient's trust and consequently, no rapport would be established between patients and nurses."

\subsubsection{Attitude}

Relevantly, one of the participants noted, "I know nurses who are not informed of their patients' medicines and only record them in nursing reports. To justify their action, they claim that the patient will ultimately die and it is better not to increase the costs."

\subsubsection{Experience}

Regarding the importance of experience in professional competence, one of the participants said, "There are a number of nursing practices, which are exclusively obtained via experience. Some examples are working in conditions such as CPR or care for a patient in shock. Most of the skills are acquired via practice and repetition, and the nurses keep going until they are experts.”

\subsubsection{Motivation}

On the importance of motivation in professional competence, one of the participants noted, "Motivation is a factor determining the quality of care for a patient. We do not expect quality care from nurses who are less motivated for this occupation or those who are financially motivated. Hence, motivation is a major factor that nursing authorities need to consider and nurture or improve it."

\subsubsection{Clinical Judgment}

Clinical judgment is another important domain in the professional competence. One of the participants stated, "Each nurse makes the best decisions based on a series of measures, including a patient's assessment, his/her own basic knowledge, experience, critical thinking, and problem-solving skills. A nurse with no ability to judge clinically is not competent in his job."

\subsubsection{Professional Independence}

Regarding the significance of professional independence, one of the participants said, "Professional independence directs nurses towards making appropriate decisions in clinical settings. A nurse with no authority to act freely and independently in terms of nursing care is not to be considered a professional competent nurse."

\subsubsection{Relationship with Patients}

Relationship with patients is another domain of professional competence in nursing. One of the participants stated, "Having appropriate communication skills in dealing with a patient is a key in making trust and, subsequently, enhancing patient satisfaction. This, in turn, would lead to a better understanding of their problems and nurse's efforts to resolve or mitigate these problems."

\subsubsection{Holistic Care}

Considering holistic care, one of the participants mentioned, "Care has a variety of physical, mental, cultural, social and environmental dimensions. A good nurse should be considerate toward all these dimensions and provide the patient with holistic care."

\subsubsection{Teamwork and Cooperation}

Regarding the cooperation and teamwork, one of the participants said, "Inter-and intra-occupation cooperation is an essential element of care provision and improves the quality of health services provided to the patient. Nowadays, nothing can be done without such communication and cooperation."

\subsubsection{Management and Leadership}

According to one participants' quote, "A nurse must also be a good manager and a good leader. A nurse is the core of the care team and always plays the role of a facilitator and coordinator. He/she should be familiar with leadership and management skills.”

\subsubsection{Moral Action}

According to another participant's quote, "Moral action has no clear definition; however, it can be said that the nurse is the patient's attorney, supporter, and family. $\mathrm{He} /$ she must always provide the best quality services with no bias and care for racial, sexual, and other debates. Conscientiousness plays a significant role here, and the nurse, having focused on the patient and the family, must have 
the power to distinguish good from the evil in order to provide moral care and obtain the patient and his family's consent."

\subsubsection{Perseverance and Persistence}

Concerned about the perseverance and persistence, one of the participants mentioned, "Perseverance and persistence, as well as great patience, is of the essence to those involved in this profession. Without these characteristics, achieving the desired goals would be impossible."

\subsubsection{Ability to Work in Difficult and Complex Situations}

According to one of the participants' quote, "Each nurse should have the ability to work in difficult and complex situations. I myself as a nurse have experienced shifts when I did not even have the opportunity to eat a bit let alone taking a rest. A person in this profession should be aware of these issues and, as a result, sensitive and weak individuals are not suitable for this profession."

\subsubsection{Creativity}

One of the participants stated, "I think a nurse without creativity acts like an iron man performing a task. Nursing art is realized by creativity, and a creative nurse finds ways to work effectively and use the minimum available resources.

\subsubsection{Responsibility}

According to one participant's quote, “A responsible nurse responds to the patient's care and aims to enhance a patient and his family's satisfaction. Each nurse is responsible for and responsive to his or her operations, and the nurse who does not take over the responsibilities is not qualified to work in this profession."

\subsection{Final Analytical Phase}

In the final analytical phase, the qualitative data obtained from the study were analyzed using the conventional content analysis. Coding was performed and the themes were extracted. Finally, the themes were compared with the findings presented at the theoretical analytical phase and the similarities and differences between the two sets of findings were determined.

The findings of the final analysis suggest that professional competence in nursing is a complex and multidimensional concept and includes physical, mental, psychological, and social dimensions. Professional competence has different domains (i.e., 16 domains based on the results of the fieldwork phase analysis).

The results obtained from the literature reviews are consistent with those of the fieldwork phase. The only difference was that the participants in the fieldwork phase introduced some aspects of professional competence in nursing, which were not well-addressed in the literature. The majority of literature defined professional competence in nursing as a combination of knowledge, skills, and attitudes, and the participants contributed to this definition to be more comprehensive.

\subsubsection{Towards a More Complete Definition of Professional Com- petence in Nursing}

According to the review of the literature and findings of the fieldwork phase, the following definition can be proposed for professional competence in nursing: "Professional competence in nursing is a complex, relative, context-dependent, and variable concept, and involves the combination of knowledge, skills, and attitudes, and the logical, scientific, and behavioral features in an individual, on the basis of which one can perform his roles professionally and independently in a standard manner and have appropriate clinical judgment in different situations."

\section{Discussion}

The findings of this hybrid analysis showed that professional competence in nursing is a complex and multidimensional concept with a variety of domains. The precise identification of these domains provides a situation for developing accurate and objective tools to assess students and nurses' competence, and thus employs qualified nurses in health care systems. These would lead to quality care, cost reduction, and enhanced satisfaction for patients, organization, and nurses.

According to the participants' quotes in this study, competence is a relative and variable concept that can be increased or decreased over time. This is also consistent with what a number of other theorists have proposed.

Giuffrida writes, "Competence is a dynamic process evolving alongside the development of an individual's experiences, knowledge, and skills throughout his/her work. In fact, the acquisition of competence is a process, not the end point (24).

Lima et al. writes, the process of professional competence acquisition connected to the concept of lifelong learning, and writes, "Professional competence is acquired and evolves over time" (25).

The participants also believed that competence is context-dependent concept and varies from one situation to another, and the context in which the nurse works in those situations should be considered. In general, based on a common perception of competence, it is a list of activities, regardless of the environment and context in which these activities should take place. In a majority of existing 
documents, however, competence has been introduced as a context-dependent concept.

Mulder writes, "Competence cannot be considered regardless of the context and the environment where it occurs, and cannot be considered independent from the context (26).

Eng and Pai write, "There are various definitions, both simple and complicated, for clinical competence in the literature". They define competence as the creation of a cognitive attribute, and interpersonal, moral, and personality skills, and emphasizes that competence is always contextoriented (27).

The findings of the literature review showed that competence includes an individual's abilities and functional features in performing tasks and enables one to conduct acceptable skills and standards in a desirable manner (28).

Further, Stevens et al. writes competence as the wise and ordinary use of knowledge, technical skills, clinical and emotional justifications, values, and reflection in daily tasks for personal and social benefits of service delivery (29).

Although there are a number of different perceptions and definitions of competence, the review of the literature suggests that knowledge, attitude, and skills are the main components of a majority of the definitions.

As indicated, the findings of the present study imply that professional competence in nursing is a complex, relative, context-dependent and variable concept, and combines an individual's knowledge, skills, attitudes, and logical, scientific, and behavioral attributes, on the basis of which one can perform his roles professionally and independently in a standard manner and have an appropriate clinical judgment in different situations.

\subsection{Implications for Practice}

Nurses' professional competence is a controversial topic in the field of health care in many countries of the world and it is important in many nursing areas such as education, practice, and nursing management (30). The development of tools based on areas of competence obtained from this study can be used to assess competence of nurses in performing their tasks. Also, nurses' awareness of their professional competence is a fundamental issue for maintaining standards of care, identifying their educational needs and their professional development, and ultimately the consequence of competence in the nursing profession is the safe practice and high-quality health care.

\subsection{Conclusions}

Given the findings of this study, professional competence is a multidimensional concept with a variety of domains. This study can pave the way for further research and contribute to the development of tools to assess competence based on the detected domains. Competence assessment is of the essence to ensure the achievement of minimum professional standards and readiness to play a role. The use of competence assessment benchmarks not only enhances nurses and managers' knowledge about the general status of nurses but also detects their skills and cognitive failures and impairments (31). Using the reflection process, the nurses get informed of their own performance and identify the strengths, abilities, and domains requiring further development. These measures ensure the quality of nursing care, safe care, the reduction of costs in health systems and, ultimately, the satisfaction of the patient, organization, and nurses (32).

\section{Acknowledgments}

The authors would like to express their gratitude to all participants in this study for their cooperation and valuable experiences.

\section{Footnotes}

Authors' Contribution: Study design: Samaneh Alizadeh, Leila Valizadeh, Vahid Zamanzadeh. Data collection: Samaneh Alizadeh, Leila Valizadeh, Vahid Zamanzadeh. Data analysis: Samaneh Alizadeh, Leila Valizadeh, Vahid Zamanzadeh. Study supervision: Leila Valizadeh, Vahid Zamanzadeh. Manuscript writing: Samaneh Alizadeh. Critical revisions for important intellectual content Samaneh Alizadeh, Leila Valizadeh, Vahid Zamanzadeh.

Conflict of Interests: No conflict of interest was declared by the authors.

Funding/Support: Tabriz University of Medical Sciences, Tabriz, Iran provided facilities and supported this project.

\section{References}

1. Warren N, Breman R, Budhathoki C, Farley J, Wilson LL. Perspectives of nursing faculty in Africa on global health nursing competencies. Nurs Outlook. 2016;64(2):179-85. doi:10.1016/j.outlook.2015.11.016. [PubMed: 26827192].

2. Pijl-Zieber EM, Barton S, Konkin J, Awosoga O, Caine V. Competence and competency-based nursing education: Finding our way through the issues. Nurse Educ Today. 2014;34(5):676-8. doi: 10.1016/j.nedt.2013.09.007. [PubMed: 24090616].

3. Tan K, Chong MC, Subramaniam P, Wong LP. The effectiveness of outcome based education on the competencies of nursing students: A systematic review. Nurse Educ Today. 2018;64:180-9. doi: 10.1016/j.nedt.2017.12.030. [PubMed: 29500999].

4. Leung K, Trevena L, Waters D. Development of a competency framework for evidence-based practice in nursing. Nurse Educ Today. 2016;39:189-96. doi: 10.1016/j.nedt.2016.01.026. [PubMed: 27006055]. 
5. Clark M, Raffray M, Hendricks K, Gagnon AJ. Global and public health core competencies for nursing education: A systematic review of essential competencies. Nurse Educ Today. 2016;40:173-80. doi: 10.1016/j.nedt.2016.02.026. [PubMed: 27125169].

6. Backhaus R, Verbeek H, van Rossum E, Capezuti E, Hamers JP. Future distinguishing competencies of baccalaureate-educated registered nurses in nursing homes. Geriatr Nurs. 2015;36(6):438-44. doi: 10.1016/j.gerinurse.2015.06.012. [PubMed: 26283584].

7. Jasemi M, Whitehead B, Habibzadeh H, Zabihi RE, Rezaie SA. Challenges in the clinical education of the nursing profession in Iran: A qualitative study. Nurse Educ Today. 2018;67:21-6. doi: 10.1016/j.nedt.2018.04.010. [PubMed: 29727826].

8. Wu FQ, Wang YL, Wu Y, Guo M. Application of nursing core competency standard education in the training of nursing undergraduates. Int J Nurs Sci. 2014;1(4):367-70. doi: 10.1016/j.ijnss.2014.10.010.

9. Bagheri Nesami M, Rafiei F, Parvizi S, Esmaeili R. [Concept analysis of competency in nursing: qualitative research and delivery of a hybrid model]. J Mazandaran Univ Med Sci. 2008;67(1). Persian.

10. McEwen M, Wills EM. Theoretical basis for nursing. Lippincott Williams \& Wilkins; 2017.

11. Holloway I, Galvin K. Qualitative research in nursing and healthcare. John Wiley \& Sons; 2016.

12. Connelly LM. Focus Groups. Medsurg Nurs. 2015;24(5):369-70. [PubMed: 26665875].

13. Doody O, Slevin E, Taggart L. Focus group interviews in nursing research: Part 1. Br J Nurs. 2013;22(1):16-9. doi: 10.12968/bjon.2013.22.1.16. [PubMed: 23299206].

14. Pai HC. The effect of a self-reflection and insight program on the nursing competence of nursing students: A longitudinal study.J Prof Nurs. 2015;31(5):424-31. doi: 10.1016/j.profnurs.2015.03.003. [PubMed: 26428348].

15. Zamanzadeh V, Roshangar F, Valizadeh L, FathiAzar E. [The challenges of newly graduated nurses for gaining competence in clinical judgment: A qualitative study]. J Urmia Nurs Midwifery Fac. 2014;12(2):11728. Persian.

16. Khomeiran RT, Yekta ZP, Kiger AM, Ahmadi F. Professional competence: Factors described by nurses as influencing their development. Int Nurs Rev. 2006;53(1):66-72. doi: 10.1111/j.1466-7657.2006.00432.x. [PubMed: 16430763].

17. Scanlon AP. An analysis of the concept of competence in nursing education. Adv Pract Nurs. 2017;2(4). doi: 10.4172/2573-0347.1000144.

18. Bowling AM, Cooper R, Kellish A, Kubin L, Smith T. No evidence to support number of clinical hours necessary for nursing competency. J Pediatr Nurs. 2018;39:27-36. doi: 10.1016/j.pedn.2017.12.012. [PubMed: 29525213].

19. Schofield R, Chircop A, Baker C, Dietrich Leurer M, Duncan S, Wotton D. Entry-to-practice public health nursing competencies: A Delphi method and knowledge translation strategy. Nurse Educ Today.
2018;65:102-7. doi: 10.1016/j.nedt.2018.03.001. [PubMed: 29547810].

20. Saleh U, O'Connor T, Afaneh T, Moore Z, Patton D, Derwin R. The use of a competence fair to validate nursing competence. Nurse Educ Today. 2017;57:1-7. doi:10.1016/j.nedt.2017.06.007. [PubMed: 28675822].

21. Blazun H, Kokol P, Vosner J. Survey on specific nursing competences: Students' perceptions. Nurse Educ Pract. 2015;15(5):359-65. doi: 10.1016/j.nepr.2015.02.002. [PubMed: 25766877].

22. Windsor C, Douglas C, Harvey T. Nursing and competencies - a natural fit: the politics of skill /competency formation in nursing. Nurs Inq. 2012;19(3):213-22. doi: 10.1111/j.1440-1800.2011.00549.x. [PubMed: 22882505].

23. Tzeng HM, Ketefian S. Demand for nursing competencies: An exploratory study in Taiwan's hospital system. J Clin Nurs. 2003;12(4):509-18. doi: 10.1046/j.1365-2702.2003.00738.x. [PubMed: 12790864].

24. [No authors listed]. Standards for continuing competence.Am JOccup Ther. 2015;69 Suppl 3:6913410055p1-3. doi: 10.5014/ajot.2015.696S16. [PubMed: 26713954].

25. Lima S, Newall F, Jordan HL, Hamilton B, Kinney S. Development of competence in the first year of graduate nursing practice: A longitudinal study. J Adv Nurs. 2016;72(4):878-88. doi: 10.1111/jan.12874. [PubMed: 26732648].

26. Mulder M. Conceptions of professional competence. International handbook of research in professional and practice-based learning. Springer; 2014. p. 107-37. doi: 10.1007/978-94-017-8902-8_5.

27. Eng CJ, Pai HC. Determinants of nursing competence of nursing students in Taiwan: The role of self-reflection and insight. Nurse Educ Today. 2015;35(3):450-5. doi: 10.1016/j.nedt.2014.11.021. [PubMed: 25534773].

28. Zamanzadeh V, Roshangar F, Fathi-Azar E, Valizadeh L, Kirkwood J. Experiences of newly graduated nurses on strategies of gaining selfconfidence during their initial work: A qualitative study. J Nurs Res. 2014;22(4):283-91. doi: 10.1097/jnr.0000000000000050. [PubMed: 25265368].

29. Stevens B, Hyde J, Knight R, Shires A, Alexander R. Competency-based training and assessment in Australian postgraduate clinical psychology education. Clin Psychol. 2017;21(3):174-85. doi: 10.1111/cp.12061.

30. Scott Tilley DD. Competency in nursing: A concept analysis. J Contin Educ Nurs. 2008;39(2):58-64. quiz 65-6, 94. doi: 10.3928/0022012420080201-12. [PubMed: 18323142].

31. Tommasini C, Dobrowolska B, Zarzycka D, Bacatum C, Bruun AM, Korsath D, et al. Competence evaluation processes for nursing students abroad: Findings from an international case study. Nurse Educ Today. 2017;51:41-7. doi: 10.1016/j.nedt.2017.01.002. [PubMed: 28122273].

32. Dellai M, Mortari L, Meretoja R. Self-assessment of nursing competencies-validation of the Finnish NCS instrument with Italian nurses. Scand J Caring Sci. 2009;23(4):783-91. doi: 10.1111/j.14716712.2008.00665.x. [PubMed:19473316]. 


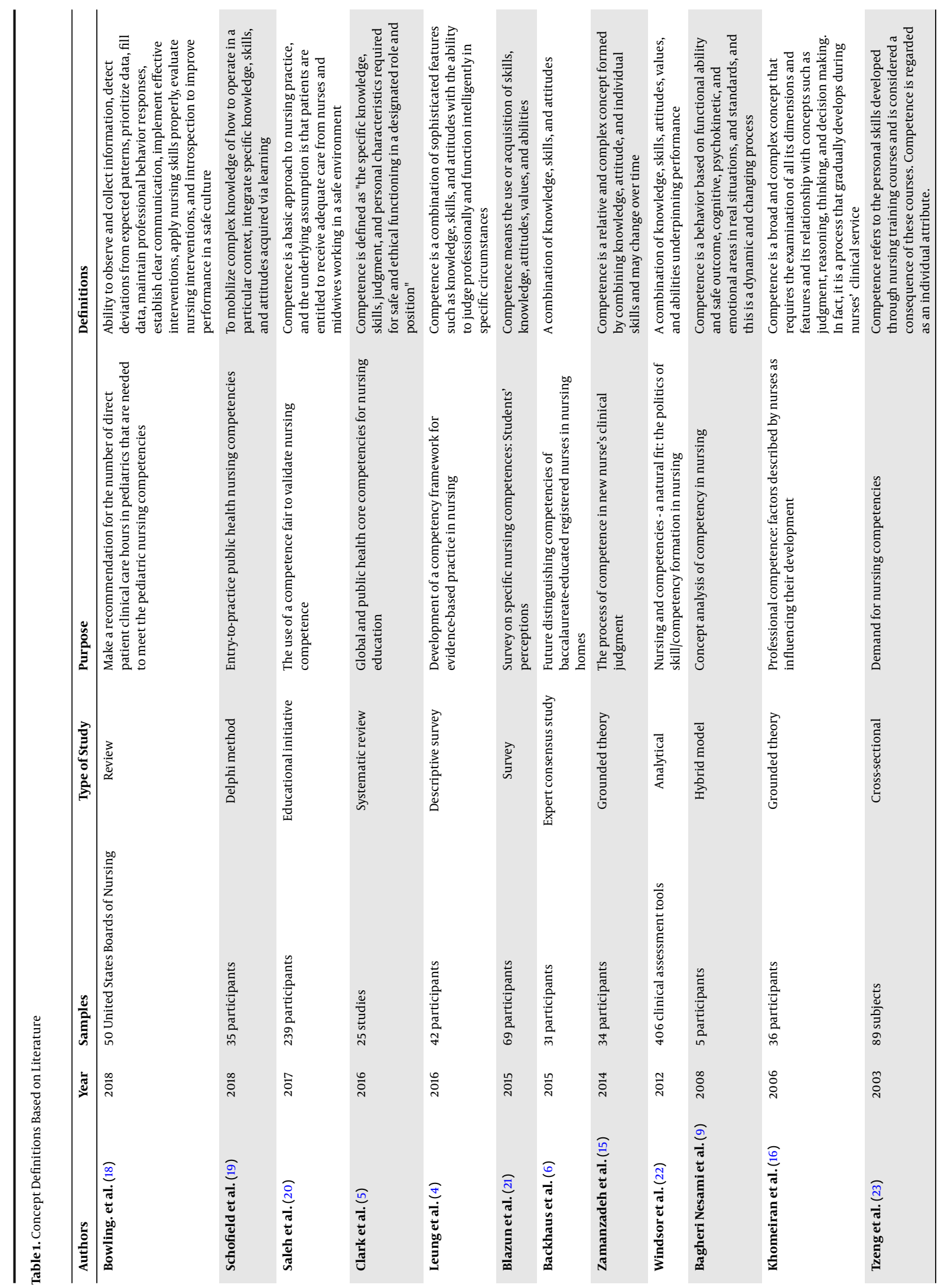

